\title{
LITAF, HHEX, and DUSP1 expression in mesenchymal stem cells from patients with psoriasis
}

\author{
W.J. Chang ${ }^{1}$, X.P. Niu', R.X. Hou' ${ }^{1}$, J.Q. Li', R.F. Liu', Q. Wang ${ }^{1}$, C.F. Wang ${ }^{2}$, \\ X.H. Li ${ }^{1}$, G.H. Yin ${ }^{1}$ and K.M. Zhang ${ }^{1}$ \\ ${ }^{1}$ Institute of Dermatology, Taiyuan City Central Hospital, Taiyuan, \\ Shanxi Province, China \\ ${ }^{2}$ Institute of Central Laboratory, Shanxi Medical University, Taiyuan, \\ Shanxi Province, China \\ Corresponding author: K.M. Zhang \\ E-mail: Zhangkaiming@sina.com
}

Genet. Mol. Res. 14 (4): 15793-15801 (2015)

Received August 4, 2015

Accepted October 25, 2015

Published December 1, 2015

DOI http://dx.doi.org/10.4238/2015.December.1.31

ABSTRACT. Psoriasis is a common chronic relapsing inflammatory skin disease, in which mesenchymal stem cells (MSCs) have been hypothesized to play an important role in abnormal localized inflammation and vascular proliferation observed in skin lesions. Previous studies have revealed abnormal gene expression patterns, DNA methylation status, and cytokine secretion of MSCs in psoriatic skin lesions, as well as some gene expression abnormalities related to inflammation and angiogenesis. We further verified the gene and protein expressions of inflammation-related lipopolysaccharide-induced tumor necrosis factor-alpha transcription factor (LITAF), dual-specificity protein phosphatase 1 (DUSP1), and angiogenesis-related hematopoietically expressed homeobox (HHEX) in MSCs derived from the skin lesions of psoriasis patients. The gene expression of LITAF, DUSP1, and HHEX in dermal MSCs was measured at the mRNA level using reverse transcription-polymerase chain reaction and the corresponding protein expression levels were analyzed by western 
blotting analysis. The gene and protein expression levels of LITAF, HHEX, and DUSP1 in dermal MSCs were significantly lower in psoriasis patients compared to controls. Amplification and western blotting results were consistent with our previously reported gene chip data. Our results suggest that dermal MSCs in psoriatic skin lesions may be involved in the development, progression, and regulation of localized inflammatory abnormalities by reducing the expression of LITAF, HHEX, and DUSP1, which are related to inflammation and angiogenesis.

Key words: Dual-specificity protein phosphatase 1; Mesenchymal stem cell; Hematopoietically expressed homeobox; Lipopolysaccharide-induced tumor necrosis factor-alpha transcription factor; Psoriasis

\section{INTRODUCTION}

Psoriasis is an immune-mediated chronic inflammatory skin disease mainly characterized by excessive proliferation of keratinocytes, infiltration of $\mathrm{T}$ cells, dendritic cells, monocytes and neutrophilic myelocytes, and proliferative expansion of superficial dermal capillaries. Although the underlying mechanism is presently unclear, substantial evidence suggests that $\mathrm{T}$ cell-based immune disorders play a key role in the pathogenesis of psoriasis (Cai et al., 2012). Such immune disorders mainly manifest as overactivation of $C D 4^{+} \mathrm{T}$ lymphocytes, joint participation of a variety of immune cells such as monocytes, dendritic cells, and B lymphocytes, and high expression of Th1 and Th17 cytokines such as interleukin (IL)-2, IL-6, IL-8, IL-22, IL-23, tumor necrosis factor- $\alpha$ (TNF- $\alpha$ ), and interferon- $\gamma$ (Loffredo et al., 2009).

Mesenchymal stem cells (MSCs) are a class of mesoderm-derived adult stem cells with high proliferation, self-renewal, and pluripotency. In addition to bone marrow, MSCs can be derived from other tissues such as fat, umbilical cord blood, and the dermis (Chunmeng and Tianmin, 2004; Rebelatto et al., 2008). MSCs play regulatory and inhibitory roles in immune responses, and thus have been increasingly used in research on immune-related diseases such as autoimmune diseases, inflammatory diseases, and graft-versus-host disease (Le et al., 2004; Nemeth et al., 2010; Gu et al., 2010).

We previously investigated the biological characteristics and related gene expression patterns of bone marrow-derived MSCs in patients with psoriasis (Zhang et al., 2010; Hou et al., 2014). In addition to reduced proliferation activity and increased spontaneous apoptosis, bone marrow-derived MSCs in psoriasis were found to be associated with high expression of stem cell factor and granulocyte-colony stimulating factor, as well as low expression of inflammatory cytokines such as IL-1, IL-3, IL-18, and TNF- $\alpha$. Moreover, differential gene expression analysis showed that the expression of genes related to immune responses, inflammation, and lymphocyte adhesion and chemotaxis in MSCs were down-regulated in psoriatic skin lesions (Zhang et al., 2010; Hou et al., 2014). Based on previous studies, we applied gene chip technology to conduct a comparative analysis of gene expression patterns in dermal MSCs (DMSCs) in skin lesions of psoriasis patients and normal skin of healthy individuals (Hou et al., 2013, 2014). Abnormalities were detected in the methylation status and expression level of a number of genes related to inflammation, immune responses, angiogenesis, and cell proliferation (Hou et al., 2013, 2014). Our previous findings suggested that MSCs in skin lesions may be involved in localized immune responses and proliferative expansion of blood vessels in psoriasis. Based on these previous findings, in 
the present study, we further analyzed the expression of inflammation-related lipopolysaccharideinduced TNF-alpha transcription factor (LITAF), hematopoietically expressed homeobox (HHEX), and angiogenesis-related dual-specificity protein phosphatase 1 (DUSP1/DSP1) at the gene and protein levels in DMSCs derived from psoriasis patients.

\section{MATERIAL AND METHODS}

\section{Patients and sample collection}

Twelve patients with psoriasis vulgaris ( 7 males and 5 females; mean \pm SD age, $37 \pm 13$ years) were enrolled in this study (Table 1). The mean Psoriasis Area and Severity Index (PASI) at the time of sample collection was $18.6 \pm 7.5$. Detailed information on the patient characteristics is shown in Table 1. Control samples were obtained from 14 healthy volunteers who agreed to undergo plastic surgery. Written informed consent was obtained from all participants, and the study protocol was approved by the Medical Ethics Committee of Taiyuan Central Hospital.

None of the 26 participants had received systemically or topical corticosteroids, biological agents, immunosuppressive agents, or ultraviolet phototherapy within 1 month before enrolment.

Skin tissues were removed from the participants and immediately used for MSC isolation.

\begin{tabular}{|c|c|c|c|c|c|}
\hline Sample & Age (years) & Gender & Illness duration & PASI & Biopsy site \\
\hline Y5 & 23 & male & 3 years & 15.8 & abdomen/center of plaque \\
\hline Y8 & 45 & female & 10 years & 27.7 & buttock/center of plaque \\
\hline Y46 & 52 & female & 8 years & 31.4 & waist/edge of plaque \\
\hline Y50 & 19 & male & 1 year & 10.4 & thigh/edge of plaque \\
\hline Y54 & 36 & male & 6 months & 16.8 & waist/edge of plaque \\
\hline Y62 & 42 & male & 6 years & 11.4 & thigh/edge of plaque \\
\hline Y64 & 34 & female & 9 years & 9.6 & waist/center of plaque \\
\hline Y65 & 21 & male & 5 months & 14.5 & dorsum/center of plaque \\
\hline Y66 & 58 & female & 23 years & 23.4 & dorsum/center of plaque \\
\hline Y68 & 54 & female & 30 years & 28.8 & arm/edge of plaque \\
\hline Y69 & 28 & male & 5 years & 14.2 & dorsum/edge of plaque \\
\hline Y73 & 33 & male & 7 years & 19.6 & abdomen/center of plaque \\
\hline
\end{tabular}

PASI = Psoriasis Area and Severity Index.

\section{Isolation and expansion of DMSCs}

DMSCs were isolated and expanded as previously described (Hou et al., 2013). Briefly, the dermis was separated from the epidermis using $0.25 \%$ dispase (Sigma-Aldrich, St. Louis, MO, USA) and subsequently digested into a monoplast suspension. After being filtered and washed with Dulbecco's modified Eagle's medium/nutrient mixture F-12 (DMEM/F12), the cells were resuspended at a concentration of $1.0 \times 10^{5} \mathrm{cells} / \mathrm{mL}$ in DMEM/F12 containing $5 \%$ fetal bovine serum, $10 \mathrm{ng} / \mathrm{mL}$ basic fibroblast growth factor (Sigma-Aldrich), $10 \mu \mathrm{L} / \mathrm{mL} \mathrm{B27}$, and $1 \%$ antibiotic mixture $\left(100 \mathrm{U} / \mathrm{mL}\right.$ penicillin and $100 \mu \mathrm{g} / \mathrm{mL}$ streptomycin sulfate) and incubated at $37^{\circ} \mathrm{C}$ in a humidified atmosphere supplemented with $5 \% \mathrm{CO}_{2}$. Non-adherent cells were removed after $72 \mathrm{~h}$, and half of the medium was changed every 3-4 days. The adherent MSCs were passaged at $80 \%$ confluence. Cell surface markers such as cluster of differentiation CD105, CD29, CD44, CD73, CD90, CD45, CD34, and human leukocyte antigen-DR (BD Biosciences, Franklin Lakes, NJ, USA) were detected using flow cytometry (Beckman Coulter, Inc., Brea, CA, USA). 


\section{Differentiation of MSCs}

\section{Osteoblast and adipocyte differentiation}

At passage 3, the cultured cells were induced to differentiate into osteoblasts and adipocytes according to published protocols (Cheng et al., 2011). DMEM without the induction factors containing only $10 \%$ fetal bovine serum was used as a negative control in the differentiation experiments.

\section{Reverse transcriptase-quantitative polymerase chain reaction (RT-qPCR)}

RNA from all 26 samples was extracted using TRIzol (Invitrogen, Carlsbad, CA, USA) and cDNA was synthesized using $4 \mu \mathrm{L}$ total RNA, $4 \mu \mathrm{L}$ 5X PrimeScript buffer, and $12 \mu \mathrm{L}$ Rasefree $\mathrm{dH}_{2} \mathrm{O}$ in a total volume of $20 \mu \mathrm{L}$ (TaKaRa, Shiga, Japan). PCR amplification was performed in a $20-\mu \mathrm{L}$ system containing $2 \mu \mathrm{L}$ cDNA, $10 \mu \mathrm{L} 2 X$ SYBR Premix EX Taq II buffer (TaKaRa), 0.4 $\mu \mathrm{L}$ Rox Reference Dye, $6 \mu \mathrm{L}$ RNase-free $\mathrm{dH}_{2} \mathrm{O}$, and $0.8 \mu \mathrm{L}$ of each $10 \mu \mathrm{M}$ primer. The thermal cycling conditions included pre-denaturation at $95^{\circ} \mathrm{C}$ for $30 \mathrm{~s}, 40$ cycles of denaturation at $95^{\circ} \mathrm{C}$ for $10 \mathrm{~s}$, and annealing at $60^{\circ} \mathrm{C}$ for $30 \mathrm{~s}$. $\beta$-actin was used as an internal control as previously reported. PCR products were identified using $2 \%$ agarose gel electrophoresis. Primer sequences and amplification product sizes are shown in Table 2.

\begin{tabular}{|c|c|c|c|}
\hline Gene & Primer sequence & Annealing temperature $\left({ }^{\circ} \mathrm{C}\right)$ & Product size (bp) \\
\hline \multirow[t]{2}{*}{$\beta$-actin } & F: 5'-CTACAATGAGCTGCGTGTGGC-3' & $60^{\circ}$ & $271^{\circ}$ \\
\hline & R: 5'-CAGGTCCAGACGCAGGATGGC-3' & & \\
\hline \multirow[t]{2}{*}{ LITAF } & F: 5'-AACATCACGCGGGCTTCTAAC-3' & $58^{\circ}$ & $182^{\circ}$ \\
\hline & R: 5'-GCATACTCGTAAAGCTGCATGTC-3' & & \\
\hline \multirow[t]{2}{*}{ HHEX } & F: 5'-GGGCCAATCCTGTCGCTTAAT-3' & $57^{\circ}$ & $81^{\circ}$ \\
\hline & R: 5'-GTTATGCGCTTGCGAACCT-3' & & \\
\hline \multirow[t]{2}{*}{ DUSP1 } & F: 5'-TGTCCGATTCATAGTCCTGTCC-3' & $58^{\circ}$ & $93^{\circ}$ \\
\hline & R: 5'-CTCACAGCGATAAGTGCCCTC-3' & & \\
\hline
\end{tabular}

\section{Western blot analysis}

DMSCs were rinsed with ice-cold phosphate-buffered saline, and total cell protein extracts were prepared using a cell lysis buffer (Beyotime Technology, Shanghai, China) containing protease inhibitors. Next, $10 \mu \mathrm{g}$ total protein from the MSCs was boiled with sample loading buffer for 5 min and then separated by $10 \%$ sodium dodecyl sulfate polyacrylamide gel electrophoresis, followed by electro-transfer to a polyvinylidene difluoride membrane (Millipore, Billerica, MA, USA). The membranes were blocked in $5 \%$ skim milk at $4^{\circ} \mathrm{C}$ overnight and then sequentially incubated with different diluted primary antibodies the next morning [(LITAF): mouse anti-human, 1:2000; (HHEX): rabbit anti-human, 1:2000; (DUSP1): rabbit anti-human, 1:1000; glyceraldehyde 3-phosphate dehydrogenase (GAPDH):mouse anti-human, 1:5000; Abcam, Cambridge, UK] at $4^{\circ} \mathrm{C}$ overnight followed by horseradish peroxidase-conjugated secondary antibodies (goat anti-mouse IgG and goat anti-rabbit IgG; Bioss, Beijing, China) at $37^{\circ} \mathrm{C}$ for $2 \mathrm{~h}$. Enhanced chemiluminescence was performed for color development and exposure. The ratio in gray scale of the objective band under test to GAPDH was calculated to semi-quantitatively analyze protein levels. 


\section{Statistical analysis}

The SPSS 13.0 software (SPSS Inc., Chicago, IL, USA) was used to perform all statistical analyses. All data are reported as means \pm SD. The independent sample $t$-test was used to compare the mean values of the 2 groups, and the level of significance was set at $P<0.05$.

\section{RESULTS}

\section{Identification of cultured DMSCs}

Consistent with our previously published data (Hou et al., 2014), the cultured cells were positive for CD105, CD29, CD44, CD73, and CD90, negative for CD45, CD34, and human leukocyte antigen-DR, showed typical fibroblast morphological characteristics, and could be induced to differentiate into both osteoblasts and adipocytes.

\section{LITAF, HHEX, and DUSP1 expression levels in DMSCs}

We previously used gene chip technology to compare gene expression patterns in MSCs between skin lesions of psoriasis patients and the normal skin of healthy individuals. In a previous study, a total of 1927 differentially expressed genes were detected, including 1090 up-regulated genes and 837 down-regulated genes. Furthermore, the expression of a number of inflammationand angiogenesis-related genes showed obvious abnormalities in the DMSCs of psoriasis skin lesions (Hou et al., 2014). Based on these preliminary results, we further analyzed the expression of 3 genes (LITAF, HHEX, and DUSP1) on the mRNA and protein levels in DMSCs of psoriasis patients.

The results of the RT-qPCR assay showed that compared with the control group, psoriasis patients had significantly down-regulated expression of LITAF $(0.52 \pm 0.15$ vs $0.79 \pm 0.27,0.66$ fold, $\mathrm{P}<0.01)$, HHEX (0.67 \pm 0.17 vs $0.99 \pm 0.38,0.67$-fold, $\mathrm{P}<0.05)$, and DUSP1 (0.70 \pm 0.19 vs $0.89 \pm 0.21,0.78$-fold, $P<0.05)$ in the DMSCs, supporting the previously reported gene chip data (Figure 1 and Table 3).

Using GAPDH as an internal reference, western blotting analysis demonstrated that compared with the control group, psoriasis patients had significantly reduced protein expression levels of LITAF $(0.31 \pm 0.11$ vs $0.45 \pm 0.17, \mathrm{P}<0.05)$, $\operatorname{HHEX}(0.25 \pm 0.09$ vs $0.45 \pm 0.19, \mathrm{P}<0.01)$, and DUSP1 $(0.19 \pm 0.10$ vs $0.36 \pm 0.15, \mathrm{P}<0.01)$ in the DMSCs, which was consistent with the results of the RT-qPCR assay (Figure 2 and Table 4).

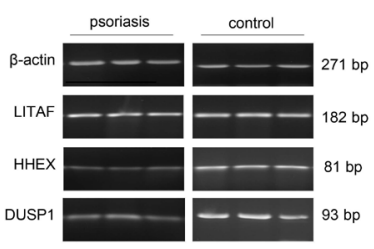

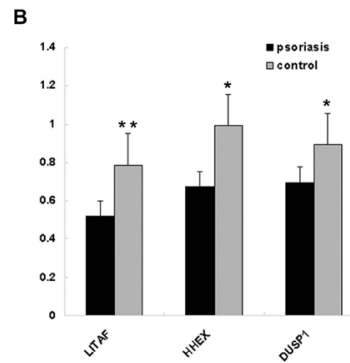

Figure 1. RT-qPCR detection of the expression of LITAF, DUSP1, and HHEX. A. mRNA expression of inflammationrelated LITAF, DUSP1, and angiogenesis-related HHEX were detected by RT-qPCR and normalized against $\beta$-actin. Comparison with healthy controls. B. Graph displays the relative mRNA expression of the different genes in the psoriasis and control groups. ${ }^{*} \mathrm{P}<0.05,{ }^{* *} \mathrm{P}<0.01$. 
Table 3. Expression level of LITAF, HHEX, and DUSP1 genes in psoriatic and normal DMSCs detected by RT-PCR.

\begin{tabular}{lcccr}
\hline Gene & Psoriasis & Control & Fold-change & P value \\
\hline LITAF & $0.52 \pm 0.15$ & $0.79 \pm 0.27$ & 0.66 & 0.005332 \\
HHEX & $0.67 \pm 0.17$ & $0.99 \pm 0.38$ & 0.67 & 0.012004 \\
DUSP1 & $0.70 \pm 0.19$ & $0.89 \pm 0.21$ & 0.78 & 0.019803 \\
\hline
\end{tabular}

Data are reported as means \pm SD.
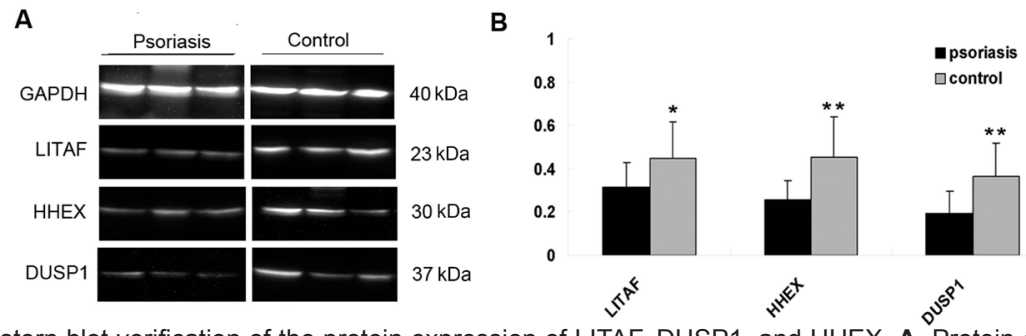

Figure 2. Western blot verification of the protein expression of LITAF, DUSP1, and HHEX. A. Protein expressions of inflammation-related LITAF, DUSP1, and angiogenesis-related HHEX were determined using western blotting and normalized against GAPDH. Comparison with healthy controls. B. Graph displays the relative protein expression of LITAF, HHEX, and DUSP1 in the psoriasis and control groups. ${ }^{*} \mathrm{P}<0.05,{ }^{* *} \mathrm{P}<0.01$.

Table 4. Expression level of LITAF, HHEX, and DUSP1 proteins in psoriasis compared to control by western blotting.

\begin{tabular}{lccc}
\hline Protein & Psoriasis & Control & P value \\
\hline LITAF & $0.31 \pm 0.11$ & $0.45 \pm 0.17$ & 0.034792 \\
HHEX & $0.25 \pm 0.09$ & $0.45 \pm 0.19$ & 0.003009 \\
DUSP1 & $0.19 \pm 0.10$ & $0.36 \pm 0.15$ & 0.004119 \\
\hline
\end{tabular}

Data are reported as means \pm SD.

\section{DISCUSSION}

Previous studies have shown that MSCs can inhibit the activation of T cells, proliferation of natural killer cells, and maturation of dendritic cells in vitro, thereby playing a negative regulatory role in the immune response (Di Nicola et al., 2002; Spaggiari et al., 2006; Nauta et al., 2006). Additionally, MSCs have been demonstrated to express vascular endothelial growth factor (VEGF), which facilitates the process of angiogenesis (Liu et al., 2011). Taking into account the functions of MSCs and the pathological features of psoriasis, we hypothesized that MSCs play an important role in the pathogenesis of this disorder.

HHEX is a transcription factor involved in the development of multiple organs, particularly of the cardiovascular system. It is encoded by the homeobox gene Hhex and is also known as proline-rich homeodomain protein $(\mathrm{PRH})$, as it contains an $\mathrm{N}$-terminal domain rich in proline residues (Noy et al., 2010). In Hhex ${ }^{\prime}$ mice, HHEX/PRH was found to bind to the promoter regions of key members of the VEGF signaling pathway, such as VEGF, VEGF receptor (VEGFR)1, VEGFR2, and neuropillin-1, inhibiting their transcription, preventing cell proliferation, and playing a negative regulatory role in angiogenesis (Noy et al., 2010). The negative regulatory effect of HHEX/PRH on angiogenesis can be blocked by casein kinase 2 (Noy et al., 2012). We previously reported that VEGF was highly expressed in DMSCs derived from psoriatic skin lesions (Hou et al., 2014) and that for the treatment of psoriasis, TNF- $\alpha$ inhibition resulted in localized inhibition of 
angiogenesis and improved skin lesion status by inhibiting VEGF, inducible nitric oxide synthase, and nitric oxide expression in DMSCs (Campanati et al., 2012). These previous findings suggest that MSCs can influence the localized proliferative expansion of blood vessels in psoriasis through the VEGF signaling pathway. In the present study, we found that reduced expression of HHEX/PRH in MSCs from psoriatic skin lesions may represent another cause of localized proliferative expansion of blood vessels.

Mitogen-activated protein kinase (MAPK) phosphatase 1, also known as DUSP1, is a protein phosphatase with dual substrate specificity that catalyzes the dephosphorylation of phosphorylated threonine/tyrosine residues. DUSP1 is widely present in different tissues and is known to regulate various cellular activities, including gene expression, cell proliferation, division, differentiation, and apoptosis (Pearson et al., 2001). Moreover, DUSP1 also specifically inhibits the activity of various MAPK family members, such as p38, c-Jun N-terminal kinase, and extracellular signalregulated kinase. It is an important regulatory factor that mediates the self-limitation and promotes the resolution of inflammation in cells. Activation of the MAPK signaling pathway is considered to be a key step in the maturation and function of immune cells. DUSP, as a specific inhibitor of the MAPK signaling pathway, can specifically degrade and inactivate MAPK (Zheng and Guan, 1993), and thus plays a key role in both autoimmunity and acquired immunity (Liu et al., 2007). In the cell, DUSP1 preferentially inhibits the p38 and c-Jun N-terminal kinase pathways rather than the extracellular signal-regulated kinase pathway (Franklin and Kraft, 1997). The extracellular signalregulated kinase 1/2 signaling pathway regulates cell growth and differentiation, whereas the c-Jun $\mathrm{N}$-terminal kinase and p38 MAPK signal transduction pathways play roles in stress responses such as inflammation and apoptosis. A variety of stimuli (e.g., mitogens, hypoxia, hormones, oxidative stress, ultraviolet radiation, and $\mathrm{Ca}^{2+}$ ) that can activate the MAPK signaling pathway can also induce the gene expression of Dusp1 (Ryser et al., 2001). In turn, DUSP1 can degrade the MAPK molecule for negative feedback inhibition of the MAPK signaling pathway, thereby inhibiting the expression of a variety of inflammatory cytokines, including IL-6, IL-8, chemokine (C-C motif) ligand 2, chemokine (C-X-C motif) ligand (CXCL)3, CXCL1, and CXCL2 (Kondoh and Nishida, 2007; Shah et al., 2014), and reducing the stimulatory effects on the body. Decreased DUSP1 expression in the DMSCs of psoriasis patients may reduce its inhibitory effects on the MAPK signaling pathway, and localized enhancement of MAPK signaling activity in skin lesions is thought to be an important factor contributing to the development of psoriasis, particularly in the early stage. Thus, DUSP1 may provide a new target for the clinical treatment of inflammation-related diseases.

TNF- $\alpha$ is a key inflammatory factor involved in the pathogenesis of psoriasis, and its expression is influenced by various factors. LITAF is a novel transcription factor that has been demonstrated to be overexpressed upon stimulation of bacterial lipopolysaccharide and phosphorylated by p38 $\alpha$ kinase. Phosphorylated LITAF can form a complex with STAT6(B), which is subsequently transferred from the cytoplasm into the nucleus, where it binds to the promoter region of lipopolysaccharide-induced genes such as TNF- $\alpha$, resulting in increased transcription of certain inflammatory cytokines such as CXCL1 (GRO), IL-1 $\alpha$, CCL5 (RANTES), TNF- $\alpha$, interferon- $\gamma$, CCL8 (MCP-2), and IL-10 (Tang et al., 2003, 2005, 2006). Accordingly, this complex plays a crucial role in the innate immune response. Furthermore, LITAF is expressed to varying degrees in different lymphoid tissues such as the peripheral blood cells, lymph nodes, and the spleen, as well as in non-lymphoid organs. However, this is the first study to report that LITAF is also expressed in the DMSCs of psoriasis patients, although at a low level. This reduced expression of LITAF in MSCs derived from psoriatic skin lesions may be caused by localized negative feedback inhibition of inflammatory factors. Alternatively, MSCs may inhibit LITAF expression during the chronic phase of the disease, thereby regulating localized expression of inflammatory factors in the skin lesions 
and further influencing the outcome of psoriasis. However, the underlying mechanisms behind this down-regulation must be verified in future studies.

In conclusion, this study revealed abnormal expression of several inflammation- and angiogenesis-related genes in DMSCs in skin lesions from patients with psoriasis. Combined with our previous findings and those of other researchers, we speculate that DMSCs play an important role in the development, progression, and outcome of psoriasis. In the early stage of the disease, MSCs may promote disease progression by promoting angiogenesis and releasing proinflammatory cytokines, as well as by inhibiting the reduction of inflammatory cytokines; conversely, in the late stage of the disease, MSCs may inhibit angiogenesis and release inflammatory factors, thereby improving the skin lesion status. Further experimental data are needed to support this hypothesis, and this will be the focus of our future research efforts.

\section{Conflicts of interest}

The authors declare no conflict of interest.

\section{ACKNOWLEDGMENTS}

Research supported by the National Natural Science Foundation of China (\#81271768 and \#81472888).

\section{REFERENCES}

Cai Yi, Fleming C and Yan J (2012). New insights of T cells in the pathogenesis of psoriasis. Cell. Mol. Immunol. 9: 302-309.

Campanati A, Orciani M, Gorbi S, Regoli F, et al. (2012). Effect of biologic therapies targeting tumour necrosis factor- $\alpha$ on cutaneous mesenchymal stem cells in psoriasis. Br. J. Dermatol. 167: 68-76.

Cheng H, Qiu L, Zhang H, Cheng M, et al. (2011). Arsenic trioxide promotes senescence and regulates the balance of adipogenic and osteogenic differentiation in human mesenchymal stem cells. Acta Biochim. Biophys. Sin. 43: 204-209.

Chunmeng S and Tianmin C (2004). Skin: a promising reservoir for adult stem cell populations. Med. Hypotheses 62: 683-688.

Di Nicola M, Carlo-Stella C, Magni M, Milanesi M, et al. (2002). Human bone marrow stromal cells suppress T-lymphocyte proliferation induced by cellular or nonspecific mitogenic stimuli. Blood 99: 3838-3843.

Franklin CC and Kraft AS (1997). Conditional expression of the mitogen-activated protein kinase (MAPK) phosphatase MKP-1 preferentially inhibits p38 MAPK and stress-activated protein kinase in U937 cells. J. Biol. Chem. 272: 16917-16923.

Gu Z, Akiyama K, Ma X, Zhang H, et al. (2010). Transplantation of umbilical cord mesenchymal stem cells alleviates lupus nephritis in MRL/pr mice. Lupus 19: 1502-1514.

Hou R, Yin G, An P, Wang C, et al. (2013). DNA methylation of dermal MSCs in psoriasis: identification of epigenetically dysregulated genes. J. Dermatol. Sci. 72: 103-109.

Hou R, Yan H, Niu X, Chang W, et al. (2014). Gene expression profile of dermal mesenchymal stem cells from patients with psoriasis. J. Eur. Acad. Dermatol. Venereol. 28: 1782-1791.

Kondoh K and Nishida E (2007). Regulation of MAP kinases by MAP kinase phosphatases. Biochim. Biophys. Acta 1773: 1227-1237.

Le Blanc K, Rasmusson I, Sundberg B, Götherström C, et al. (2004). Treatment of severe acute graft-versus-host disease with third party haploidentical mesenchymal stem cells. Lancet 363: 1439-1441.

Liu Y, Shepherd EG and Nelin LD (2007). MAPK phosphatases-regulating the immune response. Nat. Rev. Immunol. 7: 202-212.

Liu Y, Han ZP, Zhang SS, Jing YY, et al. (2011). Effects of inflammatory factors on mesenchymal stem cells and their role in the promotion of tumor angiogenesis in colon cancer. J. Biol. Chem. 286: 25007-25015.

Loffredo S, Ayala F, Marone G, Delfino G, et al. (2009). Immunopathogenesis of psoriasis and pharmacological perspectives. J. Rheumatol. 83: 9-11.

Nauta AJ, Kruisselbrink AB, Lurvink E, Willemze R, et al. (2006). Mesenchymal stem cells inhibit generation and function of both CD34+-derived and monocyte-derived dendritic cells. J. Immunol. 177: 2080-2087.

Nemeth K, Keane-Myers A, Brown JM, Metcalfe DD, et al. (2010). Bone marrow stromal cells use TGF- $\beta$ to suppress allergic 
responses in a mouse model of ragweed-induced asthma. Proc. Natl. Acad. Sci. U. S. A. 107: 5652-5657.

Noy P, Williams H, Sawasdichai A, Gaston K, et al. (2010). PRH/Hhex controls cell survival through coordinate transcriptional regulation of vascular endothelial growth factor signaling. Mol. Cell. Biol. 30: 2120-2134.

Noy P, Sawasdichai A, Jayaraman PS and Gaston K (2012). Protein kinase CK2 inactivates PRH/Hhex using multiple mechanisms to de-repress VEGF-signalling genes and promote cell survival. Nucleic Acids Res. 40: 9008-9020.

Pearson G, Robinson F, Beers Gibson T, Xu BE, et al. (2001). Mitogen-activated protein (MAP) kinase pathways: regulation and physiological functions. Endocr. Rev. 22: 153-183.

Rebelatto CK, Aguiar AM, Moretão MP, Senegaglia AC, et al. (2008). Dissimilar differentiation of mesenchymal stem cells from bone marrow, umbilical cord blood, and adipose tissue. Exp. Biol. Med. 233: 901-913.

Ryser S, Tortola S, van Haasteren G, Muda M, et al. (2001). MAP kinase phosphatase-1 gene transcription in rat neuroendocrine cells is modulated by a calcium-sensitive block to elongation in the first exon. J. Biol. Chem. 276: 33319-33327.

Shah S, King EM, Chandrasekhar A and Newton R (2014). Roles for the mitogen-activated protein kinase (MAPK) phosphatase, DUSP1, in feedback control of inflammatory gene expression and repression by dexamethasone. J. Biol. Chem. 289: 13667-13679.

Spaggiari GM, Capobianco A, Becchetti S, Mingari MC, et al. (2006). Mesenchymal stem cell-natural killer cell interactions: evidence that activated NK cells are capable of killing MSCs, whereas MSCs can inhibit IL-2-induced NK-cell proliferation. Blood 107: 1484-1490.

Tang X, Fenton MJ and Amar S (2003). Identification and functional characterization of a novel binding site on TNF- $\alpha$ promoter. Proc. Natl. Acad. Sci. U. S. A. 100: 4096-4101.

Tang X, Marciano DL, Leeman SE and Amar S (2005). LPS induces the interaction of a transcription factor, LPS-induced TNFalpha factor, and STAT6 (B) with effects on multiple cytokines. Proc. Natl. Acad. Sci. U. S. A. 102: 5132-5137.

Tang X, Metzger D, Leeman S and Amar S (2006). LPS-induced TNF-alpha factor (LITAF)-deficient mice express reduced LPS-induced cytokine: Evidence for LITAF-dependent LPS signaling pathways. Proc. Natl. Acad. Sci. U. S. A. 103: 13777-13782.

Zhang K, Liu R, Yin G, Li X, et al. (2010). Differential cytokine secretion of cultured bone marrow stromal cells from patients with psoriasis and healthy volunteers. Eur. J. Dermatol. 20: 49-53.

Zheng CF and Guan KL (1993). Dephosphorylation and inactivation of the mitogen-activated protein kinase by a mitogeninduced Thr/Tyr protein phosphatase. J. Biol. Chem. 268: 16116-16119. 